\title{
Multiple vascular malformation in gut with coronary artery aneurysm: new syndrome?
}

\author{
Qian-Qian Chen and Xiu-Li Zhang* \\ Department of Gastroenterology, Chinese PLA General Hospital, Beijing, China
}

\begin{abstract}
We report a case of multiple vascular malformation in gut with coronary artery aneurysm. We discussed this case with seriousness and consulted related literatures. Finally, we couldn't diagnose it as any disease at present and considered it could be a new syndrome.
\end{abstract}

\section{Case}

A 51-year-old man was admitted to our department in March 17th, 2016, because of hematochezia. 5 months ago, he began to pass bloody stool intermittently, 5-7 times daily in the most serious period, with abdominal distention and anemia. He visited many hospitals for diagnosis and treatment. This symptom could be alleviated by hemostasis and blood transfusion. During disease, he had no abdominal pain, fever, night sweet and haematemesis. He had a history of hypertension, myocardial infarction, giant aneurysm and intramural aneurysm. He denied use of aspirin, non-steroidal and anti-inflammatory drugs. He also has a personal history of smook and drink for many years. His family history was farther, and brother died of heart disease, and cerebral hemorrhage. After admitted, his routine laboratory data were mostly normal, except microcytic hypochromic anemia (Hb 63g/L, RBC 3.1×10 $12 / \mathrm{L}$, MCV 69.7pg, MCH 20fl, MCHC $292 \mathrm{~g} / \mathrm{l}$ ) and Feces Occult Blood positive. Physical examination showed pale appearance, cardiomegaly, no haemangiomas on the skin and mostly normal in abdomen. Colonoscopy result (Figure 1), which performing to hepatic flexure (because of unbearable pain), showed multiple varices of uneven size in the colon, multiple veinal protuberant lesions under descent colon. Colon EUS result (Figure 2) showed abnormal vein in sigmoid colon which were Capsule endoscopy (Figure 3) result showed blue coarse vein were seen in proximal intestine which were different to colon lesions. Enhanced abdominal CT showed: First, multiple hypo vascular lesions in the liver and spleen (considered benign cyst possible), abnormal enhanced lesions in the left medial segment and communicating to left portal vein and middle hepatic vein (consider angiodysplasia possible); Second, multiple hypo vascular lesions in the abdomen, retro-peritoneum and mesenterium, which considered benign and lymph node reactive proliferations possible, but could not eliminate tumor or vascular anomalies; Third, abnormal enhanced lesions (varices) in the colon submucosa, abnormal density (aneurysm) and pericardial effusion. CTA (Figure 4) showed: First, aneurysm in the right and left anterior descending coronary artery, myocardial infarction in the cardiac apex and pericardial effusion; Second, angiomatosis in the colon, abdominal cavity and retroperitoneum; Third, superior and inferior mesenteric artery enlarged, and inferior mesenteric vein enlarged, and collateral circulation formed. We diagnosed as multiple vascular malformation in gut and coronary artery aneurysm at present.
This disease could be differential diagnosed from blue rubber bleb nevus syndrome (BRBNS). As usual, BRBNS is a rare disease with main features of vascular malformations in the skin and gut. The initial symptoms were mostly recurrent gastrointestinal hemorrhage and anemia, which is similar to this case. But the characteristics of vascular malformations were different from this case which is bluish, soft, painless, irregular and cavernous lesions made it easily compressible and promptly refilled after releasing pressure [1]. BRBNS might be

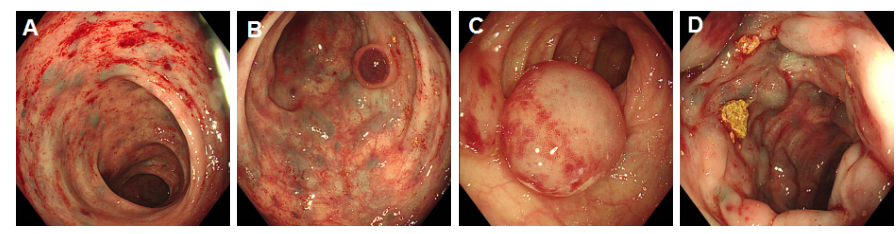

Figure 1. A. Scattered protuberant vein in rectum. B: Tiny varices and small phlebangioma in sigmoid colon. C: Huge phlebangioma in transverse colon. D: Thicker varices in transverse colon.
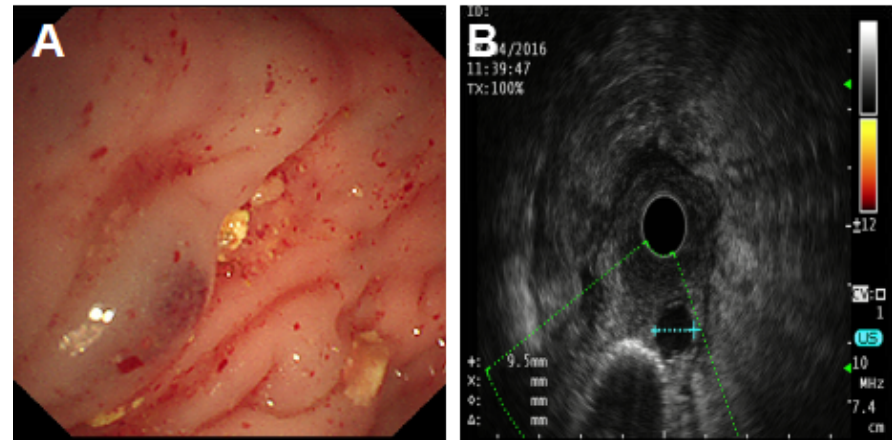

Figure 2. A: Hemispherical protrusive lesions. B: Even hypoechoic nodules in the third layer.

Correspondence to: Xiu-Li Zhang, $\mathrm{PhD}$, Department of Gastroenterology, Chinese PLA General Hospital, Beijing 100853, China, Tel: +86-10-55400305; Fax: +86-10-55400305; E-mail: zhangxl70@126.com

Key words: vascular malformation, gut, coronary artery aneurysm, new syndrome

Received: March 05, 2018; Accepted: March 15, 2018; Published: March 21, 2018 

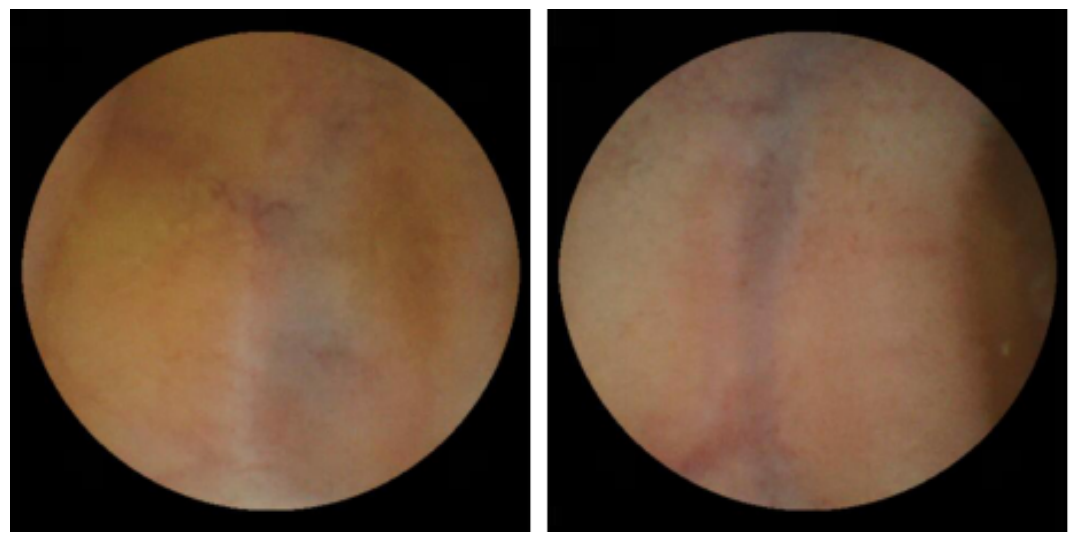

Figure 3. Blue coarse vein in proximal intestine.
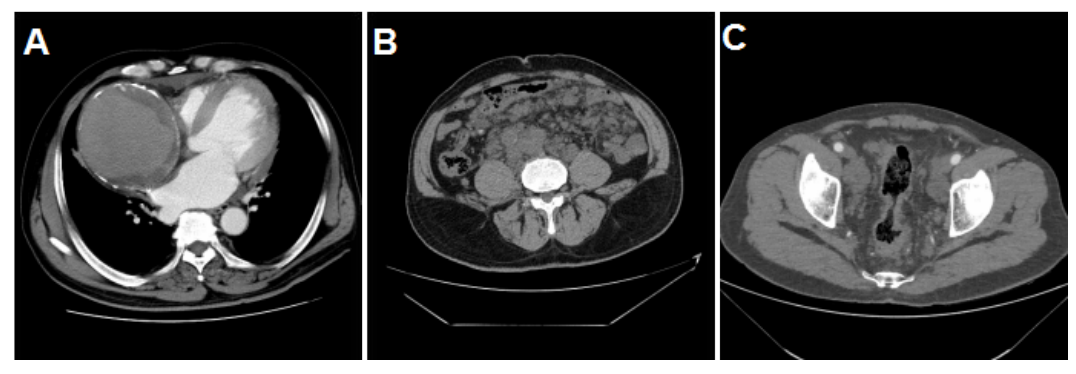

Figure 4. CTA showed multiple vascular malformation. A: Aneurysm in the right anterior descending coronary artery. B: Angiomatosis in the colon. C: Multiple abnormal veins in abdominal cavity.

involved in other organs, for example, oronasopharynx, liver, spleen, heart, lung, pleura, peritoneum, kidney, thyroid, parotid, skeletal muscle, bone, bladder, brain, penis, vulva and eyes [1]. Balato et al [3], published a case report of blue rubber bleb naevus with ateriovenous aneurysm. But is different to this case. Other differential diagnoses including Mafucci's syndrome and Rendu-Osler-Weber syndrome could also be eliminated. Therefore, we boldly speculated this case to be a new syndrome of multiple vascular malformation in gut and coronary artery aneurysm.

\section{References}

1. Aroor S, Varma C, Mundkur SC (2012) Blue Rubber-Bleb Nevus Syndrome which was Associated with an Atrial Septal Defect: A Case Report. J Clin Diagn Res 6: 15661567. [Crossref]

2. Carvalho S, Barbosa V, Santos N, Machado E (2003) Blue rubber-bleb nevus syndrome: report of a familial case with a dural arteriovenous fistula. AJNR Am J Neuroradiol 24: 1916-1918. [Crossref]

3. Balato N, Montesano M, Lembo G (1986) Blue rubber bleb naevus with arteriovenous aneurysm. Ann Dermatol Venereol 113: 245-247. [Crossref]

Copyright: $\odot 2018$ Chen QQ. This is an open-access article distributed under the terms of the Creative Commons Attribution License, which permits unrestricted use, distribution, and reproduction in any medium, provided the original author and source are credited. 